\title{
Arduino based Home Automation System: A Survey
}

\author{
Harish lyer \\ Department of Information \\ Technology \\ Dr D Y Patil Institute of \\ Engg and Tech \\ Talegaon, Pune
}

\author{
Chetan Wagaj \\ Department of Information \\ Technology \\ Dr D Y Patil Institute of \\ Engg and Tech \\ Talegaon, Pune
}

\author{
Niketan Newale \\ Department of Information \\ Technology \\ Dr D Y Patil Institute of \\ Engg and Tech \\ Talegaon, Pune
}

\author{
Ganesh Mhatre \\ Department of Information \\ Technology \\ Dr D Y Patil Institute of \\ Engg and Tech \\ Talegaon, Pune
}

\begin{abstract}
The rapidly advancing mobile communication technology and the decrease in costs make it possible to incorporate mobile technology into home automation systems. We propose a mobile and internet based home automation system that consists of a mobile phone with android capabilities, an internet based application, and a home server. The home appliances are controlled by the Arduino which receives commands from the server computer, which operates according to the commands received from the mobile application via the wireless network or the internet. In our proposed system the home server is built upon a Wi-Fi technology which receives commands from the client and the command is processed via Arduino, which allows a user to control and monitor any parameters related to the home by using any Java capable cell phone or via the internet. This paper presents an innovative low cost design and implementation of automated ambience control using user mood or based on weather conditions, interactive voice based response, appliance control, and, home security together with the design of android application to enable the cell phone to send commands and receive alerts through the server.
\end{abstract}

\section{General Terms}

Automation System, Micro-controller.

\section{Keywords}

Automation, PIC, home server, voice based response, appliance control, lights control, mobile controlled, fire sensor.

\section{INTRODUCTION}

Today's world is digitized. Starting from our handheld devices to computers to smart appliances, our world is digitized. Thus a smart home would be the next step for a better future. The system efficiently controls the lights and appliances, thus minimizing power consumption. The system makes use of the internet to control the house appliances and the lights. Apart from merely switching off and switching on the lights, the system can control it efficiently using ambient light sensors and can adjust the lights setting of the home. It enables the user to control various aspects of their appliances from a remote location through a hand held device. It hence makes a versatile system which expands the mobility of the user. The system emphasizes on controlling lights based on human mood, weather or lighting conditions which gives the user a diverse range of controlling options.

\section{VARIOUS CATEGORIES OF HOME AUTOMATION}

Over the year, home automation has grown to a new level with new and more advanced technology and integrated circuits achieving more complex functionalities. From the very basic implementation of the home automation system to the modern implementation using advance technologies and artificial intelligence, we have progressed in the field of computers being capable of making decisions which favor humans.

This implementation guarantees low cost implementation of the home automation with minimal hardware interface and maximum throughput.

\subsection{ZigBee based Automation}

ZigBee is a new hardware device that connects to various device wirelessly and reduces wiring overhead. The program is fed to the ZigBee's mcu and based on the input received by the user the device sends commands to various subsystems which controls the necessary appliance.

Also ZigBee offers low power solution for automation products. Thus, being power efficient. It can receive signals wirelessly from a device and act accordingly. Multiple hardware components can be connected which then helps in monitoring a house completely.

\subsection{Automation using PLC and Energy Conservation Methodology}

The PLC (Power Line Conservation) connects multiple equipment's at a time and monitors it carefully. If attached with a smart power module it saves a lot of energy. This research has a success rate of $90 \%$ which helps us easily track our appliance status without being physically present.

The energy conservation methodology allows only the necessary power required by the system to run the home and thus saving a lot of electricity.

\subsection{Home Automation using a Universal Remote Control}

There has been a huge inconvenience in controlling each digital home appliance which requires its own remote controller. This implements a universal remote control which is an advanced universal remote controller (URC) with the total solution for home automation and security. All kinds of appliances used in home can be controlled with the URC, which can be also connected to a computer which has an Internet access. To use the URC, it need several receivers with wired or wireless communication methods to be connected to all appliances. The receivers have many channels and IDs to control many appliances at the same time. This implementation can be applied to the automated control of all kinds of appliances installed within buildings for companies, homes, malls, hospitals, schools etc. 


\subsection{Home Automation based on 3D Virtual World}

Home automation is a system to control appliances or things in the home such as a door, lights, surveillance cameras and other electronics. A user can control a various home devices with the help of home automation system. To provide mobility, a mobile-based home automation has been developed. Controlling and monitoring are possible with a phone. But the home automations mentioned above lack user-friendliness, neither intuitive nor realistic. To improve the interface of the user, 3D virtual world is adopted as the control interface for the home automation. With the help of 3D virtual world, a user can monitor the status of home devices and control them intuitively anywhere and anytime through the internet.

\subsection{SMS based Home Automation System}

Home automation using sms based technology is a way in which we use gsm technology to communicate with the home server. The sms can be sent from any gsm based phone and the gsm module can be used to receive the sms. The gsm module is connected with the microcontroller which in turn drives various home appliances. This communication is serial and RS-232 is used for the serial communication. The system authenticates the validity of the user by accepting username and password as a new text message. Once the user is authenticated the user can then send text messages to appropriately control various home parameters. The drawback of the system is that it uses gsm technology in this fast pace world where internet has taken over most form of communication. The sms is subjected to the carrier and in case the user runs out of balance or moves to a different state where roaming charges are applicable extra, it would add to the cost of the user. The systems advantage is the gsm itself as we do not require an internet connection but an interactive user experience cannot be achieved.

\subsection{Home Automation System using Arduino and Mobile Phone}

Home Automation has made advancements in the recent years with more and more people opting for automation to simplify their lives. The appliances are controlled using Arduino which receives commands from a server computer. The server computer receives commands from an android capable mobile. The Arduino has various sensors attached which acts as the senses for the system and helps is conserving energy with smart decision making capabilities.

The system itself is modularized which helps is adding various features to the system with ease and in low time with high efficiency. The main objective of the project is to achieve automation in low costs with high level of reliability in the system.

The system receives input or commands from an android phone which is basically the user. The user has extensive control of how to operate the lights and the appliances. A new mood based lights controlling mechanism is being implemented which adjusts the lighting of the house according to the user's mood, which can be beneficial in understanding the effects of light on humans. Also, the system can adjust the lights based on weather inputs or lighting condition which helps in minimizing power consumption and helping our home being lit bright through the day. The rooms are fitted with PIR sensors which detects human presence and in case if the there are no person in a particular room, the system would shut of all the lights and necessary appliance. Thus, conserving energy.

The system can be used to control appliances which the user can control to turn on the appliance and to turn off the appliance, which helps in monitoring appliance remotely and maximizing efficiency. Additional fire detecting sensors are used to monitor any fire in the home which would immediately sound of an alarm and trip the home's main power supply.

\section{ARCHITECTURE}

The proposed system is controlled by a central computer which is attached with the microcontroller and then it control the appliances. The architecture below describes the same. The server receives signals or commands from a hand held mobile device running on android.

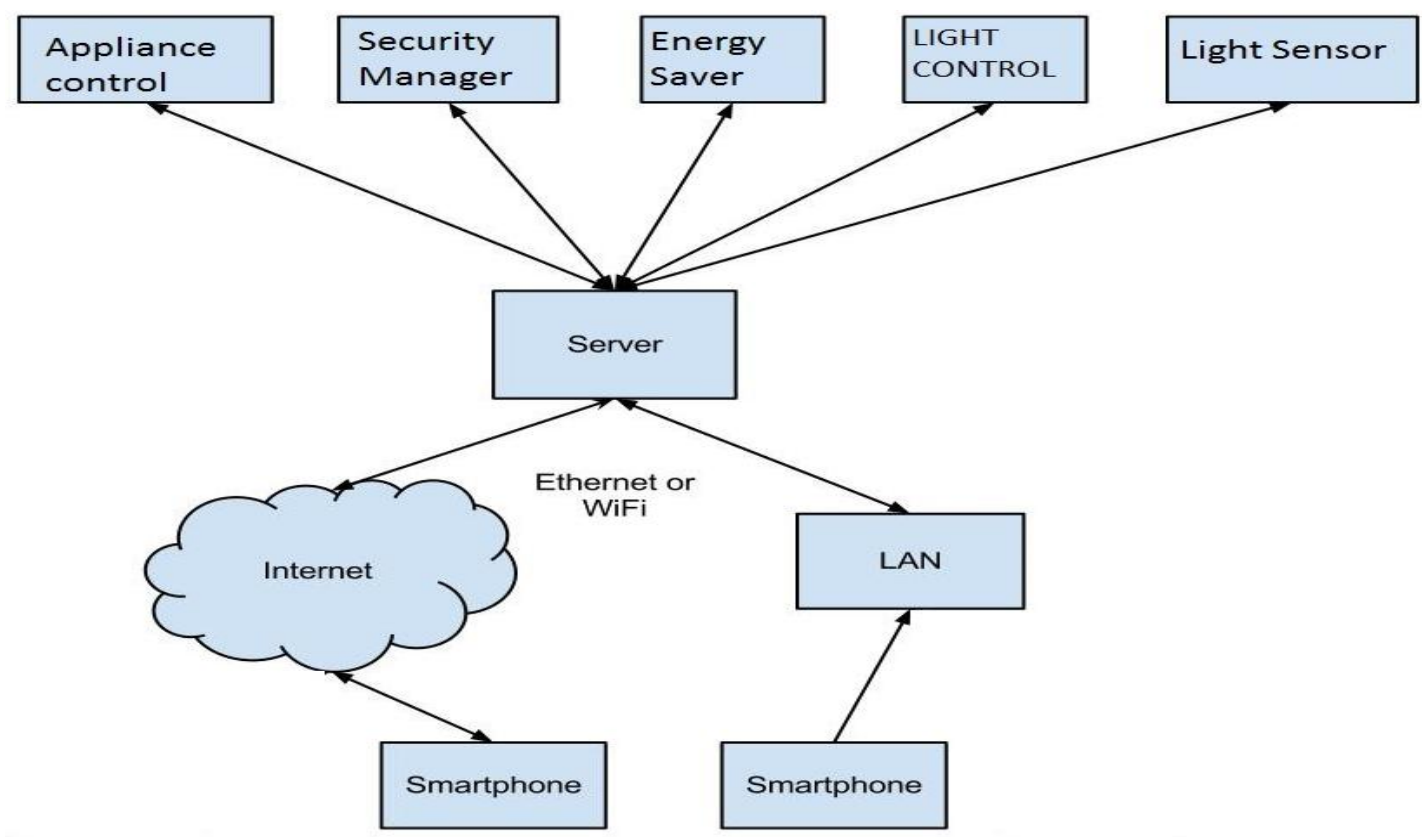

Fig 1: Architecture of the proposed Home Automation System 


\section{ACKNOWLEDGMENTS}

We are very thankful to our guide Mr Anirban Datta and Head of Information Technology Department Prof. Amol Jadhao of Dr D Y Partil Institute of Engineering and Technology Pune, Maharashtra, India for their kind support and constant encouragement for this work

\section{CONCLUSION}

In this paper we present a survey for different types of home automation system. This proposed system has a vast scope and a limitless application and if implemented to its full functionality can help minimize energy consumption. Also this project can be used to understand the effect of lights on human which in turn can help us build a smart home in near future. The system can be modularized and multiple other components can be attached to the system which will help us achieve complex functionalities. The system itself would be a smart artificial brain which would then help us in making an energy efficient world with low costs. This system in a future perspective can be used to design an interactive home automation system wherein the system will respond to human speech making it more realistic to interact with your home. It can extend a security feature which can use openCV to detect hand motions, which in emergency may be used to turn on a remote alarm. Also the system can be made to adapt to environmental changes and take necessary decisions based on the external conditions. Also, with the use of artificial intelligence we can make the system learn the changes that needs to be adapted in a particular situation. Thus, making the system completely reliable and managing a hi-tech home with least efforts.

\section{REFERENCES}

[1] Jinsoo Han; Chang-sic Choi; Wan-Ki Park; Ilwoo Lee; Sang-Ha Kim, "Smart home energy management system including renewable energy based on ZigBee and PLC,"
[2] Baraka, K.; Ghobril, M.; Malek, S.; Kanj, R.; Kayssi, A., "Low Cost Arduino/Android-Based Energy-Efficient Home Automation System with Smart Task Scheduling," Tavel, P. 2007 Modeling and Simulation Design. AK Peters Ltd.

[3] Sannella, M. J. 1994 Constraint Satisfaction and Debugging for Interactive User Interfaces. Doctoral Thesis. UMI Order Number: UMI Order No. GAX9509398., University of Washington.

[4] Saha, A.; Kuzlu, M.; Pipattanasomporn, M., "Demonstration of a home energy management system with smart thermostat control," Innovative Smart Grid Technologies (ISGT).

[5] Gupta, A.; Mudgal, A.; Jayaraj, C.; Kulkarni, J.; Jain, K.; Murarka, P.; Gujar, S., "Smart home device and energy management systems,".

[6] Taewan Kim; Hakjoon Lee; Yunmo Chung, "Advanced universal remote controller for home automation and security,"

[7] Jinsoo Han; Jaekwan Yun; Jonghyun Jang; Kwang-Roh Park, "User-friendly home automation based on 3D virtual world,"

[8] Low Cost Smart Home Automation via Microsoft Speech Recognition Md. Raihaan Kamarudin., Md. Aiman F. Md. Yusof.

[9] Embedded Web Server for Home Appliances Mr. Abhishek Vichare, Ms. Shilpa Verma.

[10] Elkamchouchi, H.; ElShafee, A., "Design and prototype implementation of SMS based home automation system," Electronics Design, Systems and Applications. 